\title{
DIGITALCOMMONS
}

\section{A Fano-Huffman Based Statistical Coding Method}

\author{
Aladdin Shamilov \\ Anadolu University, Turkey \\ Senay Asma \\ Anadolu University, Turkey
}

Follow this and additional works at: http:// digitalcommons.wayne.edu/jmasm

Part of the Applied Statistics Commons, Social and Behavioral Sciences Commons, and the Statistical Theory Commons

\section{Recommended Citation}

Shamilov, Aladdin and Asma, Senay (2007) "A Fano-Huffman Based Statistical Coding Method," Journal of Modern Applied Statistical Methods: Vol. 6 : Iss. 1 , Article 25.

DOI: $10.22237 /$ jmasm/1177993440

Available at: http://digitalcommons.wayne.edu/jmasm/vol6/iss1/25 


\title{
A Fano-Huffman Based Statistical Coding Method
}

\author{
Aladdin Shamilov Senay Asma \\ Anadolu University, Turkey
}

Statistical coding techniques have been used for lossless statistical data compression, applying methods such as Ordinary, Shannon, Fano, Enhanced Fano, Huffman and Shannon-Fano-Elias coding methods. A new and improved coding method is presented, the Fano-Huffman Based Statistical Coding Method. It holds the advantages of both the Fano and Huffman coding methods. It is more easily applicable than the Huffman coding methods and it is more optimal than Fano coding method. The optimality with respect to the other methods is realized on the basis of English, German, Turkish, French, Russian and Spanish.

Key words: Fano-Huffman based statistical coding method, probability distribution of language, entropy, information, optimal code.

\section{Introduction}

Problem Statement

Huffman's algorithm is a well-known encoding method that generates an optimal prefix encoding scheme, in the sense that the average code word length is minimum. As opposed to this, Fano's method has not been used so much because it generates prefix encoding schemes that can be sub-optimal (Rueda \& Oommen, 2004).

In this article, an improved coding method is presented, which has been named the Fano-Huffman Based Statistical Coding method and applications of this method. This method holds the both advantages of Fano and Huffman coding method. So, it is more easily applicable than the Huffman coding method and is more optimal than Fano coding method. The optimality of the mentioned coding method with

Aladdin Shamilov is a Professor in the Department of Statistics. Email: asamilov@anadolu.edu.tr. Senay Asma is a Research Assistant in the Department of Statistics. Email: senayyolacan@anadolu.edu.tr. respect to the other coding methods is realized on the basis of English, German, Turkish, French, Russian and Spanish.

The classical coding methods and the concept of optimality are described in the section titled Classical Coding Methods and Optimality.

An improved coding method, FanoHuffman Based Coding Method by which encoding schemes, which are arbitrarily close to the optimum, can be easily constructed, is introduced in the section called Fano-Huffman Based Statistical Coding Method.

In the following section, the tables of constructed binary codes are given and comparisons of considered methods in sense of optimality are made.

In the conclusion, the interpretation of optimality of these results is made subject to classical coding methods and suggestions are given.

\section{Overview}

Assume that a source alphabet, $S=\left\{s_{1}, s_{2}, \ldots s_{n}\right\}$, whose probabilities of occurrence are $\mathrm{P}=\left\{\mathrm{p}_{1}, \mathrm{p}_{2}, \ldots, \mathrm{p}_{\mathrm{n}}\right\}$, and a code alphabet, $A=\left\{a_{1}, a_{2}, \ldots a_{r}\right\}$ is given. The propose of this study is the generation of an encoding scheme, $\left\{s_{i} \rightarrow w_{i}\right\}$, in such a way 
that $\bar{l}=\sum_{i=1}^{n} p_{i} l_{i}$ is minimized, where $l_{i}$ is the length of $w_{i}$.

Information theory has important applications in probability theory, statistics and communication systems. Lossless encoding methods used to solve this problem include Huffman's algorithm (Huffman, 1952), Shannon's method (Shannon \& Weaver, 1949), arithmetic coding (Sayood, 2000), Fano's method (Hankerson, Harris, \& Johnson, 1998), enhanced Fano-based coding algorithm (Rueda $\&$ Oomen, 2004) etc. Adaptive versions of these methods have been proposed, and can be found in (Faller, 1973; Gallager, 1978; Hankerson et al., 1998; Knuth, 1985; Rueda, 2002; Sayood, 2000). The survey is necessarily brief as this is a well-reputed field.

Also, assume that the source is memoryless or zeroth-order, which means that the occurrence of the next symbol is independent of any other symbol that has occurred previously. Higher-order models include Markov models (Hankerson et al., 1998), dictionary techniques (Ziv \& Lempel, 1977; Ziv \& Lempel, 1978), prediction with partial matching (Witten, Moffat, \& Bell, 1999), grammar based compression (Kieffer \& Yang, 2000), etc., and the techniques introduced here are also readily applicable for such structure models.

\section{Classical Coding Methods and Optimality}

In this section, the fundamental steps of classical coding methods are described and the concept of optimality of codes is expounded.

\section{Classical coding methods}

Suppose that source alphabet (alphabet of language) $\mathrm{S}=\left\{\mathrm{S}_{1}, \mathrm{~S}_{2}, \ldots, \mathrm{S}_{\mathrm{n}}\right\}$ and its probability distribution $\mathrm{P}=\left\{\mathrm{p}_{1}, \mathrm{p}_{2}, \ldots, \mathrm{p}_{\mathrm{n}}\right\}$ are given.

\section{Ordinary Coding Method}

This method requires the following steps:

(a) Determine number $\ell$ satisfying the inequality $\ell \geq \log _{2} \mathrm{~N}$, where $\ell$ is the length of codeword and $\mathrm{N}$ is the the number of symbols in source alphabet;

(b) frequency;

Enumerate letter ignoring the

(c)

Convert numbers determined by

(b) from base 10 to base 2 such that $\ell$ is the length of converted number (Roman, 1997).

\section{Shannon Coding Method \\ Construction of Shannon Codes is provided by steps:} Put $\quad P=\left\{p_{1}, p_{2}, \ldots, p_{n}\right\} \quad$ in ascending order $p_{1} \geq p_{2} \geq \ldots \geq p_{n}$; Calculate $\ell_{\mathrm{i}}=\left\lceil\log _{2} \mathrm{p}_{\mathrm{i}}^{-1}\right\rceil$ the length of codeword, $i=1,2, \ldots, n$; $\mathrm{F}_{1}=0 \quad$ and $\quad \mathrm{F}_{\mathrm{k}}=\sum_{\mathrm{i}=1}^{\mathrm{k}-1} \mathrm{p}_{\mathrm{i}}, \quad 2 \leq \mathrm{k} \leq \mathrm{n}$. Then calculate $F_{i}, i=1,2, \ldots, n$; Convert dyadic fraction $F_{i}$ to binary form by using Koblitz's trick, then select first $\ell_{\mathrm{i}}$ bits as a code corresponding to $\mathrm{s}_{\mathrm{i}}$ (Hankerson et. al., 2003).

Fano Coding Method This method involves the steps:

(a) Perform the probabilities of symbols in source alphabet in ascending order $\mathrm{p}_{1} \geq \mathrm{p}_{2} \geq \ldots \geq \mathrm{p}_{\mathrm{n}}$

(b)

Divide the set of symbols into two subsets such that the sum of the probabilities of occurrences of symbols in each subset are equal or almost equal. Then, assign a 0 to first subset and a 1 to second;

(c) Repeat step (a) until all subsets have a single element (Венцель, 1969). 
Enhanced Fano Coding Method steps:

This method proposed the following

(a) Consider the source alphabet $\mathrm{S}=\left\{\mathrm{s}_{1}, \mathrm{~s}_{2}, \ldots, \mathrm{s}_{\mathrm{n}}\right\} \quad$ whose probability distribution of occurrences is $\mathrm{P}=\left\{\mathrm{p}_{1}, \mathrm{p}_{2}, \ldots, \mathrm{p}_{\mathrm{n}}\right\}$, where $\mathrm{p}_{1} \geq \mathrm{p}_{2} \geq \ldots \geq \mathrm{p}_{\mathrm{n}} ;$

(b) Obtain $\phi: \mathrm{s}_{1} \rightarrow \mathrm{w}_{1}, \ldots, \mathrm{s}_{\mathrm{n}} \rightarrow \mathrm{w}_{\mathrm{n}}$ the encoding scheme by Fano's method;

(c) Rearrange $\mathrm{w}_{1}, \mathrm{w}_{2}, \ldots, \mathrm{w}_{\mathrm{n}}$ into $\mathrm{w}_{1}^{\prime}, \mathrm{w}_{2}^{\prime}, \ldots, \mathrm{w}_{\mathrm{n}}^{\prime}$ such that $\ell_{\mathrm{i}}^{\prime} \leq \ell_{\mathrm{j}}^{\prime}$ for all $\mathrm{i}<\mathrm{j}$, and simultaneously maintain $\mathrm{s}_{1}, \mathrm{~s}_{2}, \ldots, \mathrm{s}_{\mathrm{n}}$ in the same order, to yield the encoding scheme: $\phi^{\prime}: \mathrm{s}_{1} \rightarrow \mathrm{w}_{1}^{\prime}, \ldots, \mathrm{s}_{\mathrm{n}} \rightarrow \mathrm{w}_{\mathrm{n}}^{\prime} \quad$ (Rueda \& Oommen, 2004).

\section{Huffman Coding Method}

This method is bottom-up while the others are top-down. It can be explained more clearly as follows:

(a) Sort symbols of source alphabet in decreasing order of their probabilities;

(b) Merge the two least-probable letter into a single output whose probability is the sum of the corresponding probabilities;

(c) Go to step (a) if the number of remaining outputs is more than 2 ;

(d) Assign a 0 and a 1 arbitrarily as code words for the two remaining outputs;

(e) Append the current codeword with a 0 and a 1 to obtain the codeword the preceding outputs and repeat step (e) if an output is the result of the merger of two outputs in a preceding step. Stop if no output is preceded by another output in a preceding step (Aazhang, 2004).

Shannon-Fano-Elias Coding Method

This method can be explained by steps: (a) Perform the source alphabet $\mathrm{S}=\left\{\mathrm{S}_{1}, \mathrm{~s}_{2}, \ldots, \mathrm{S}_{\mathrm{n}}\right\} \quad$ whose probability distribution of occurrences is $\mathrm{P}=\left\{\mathrm{p}_{1}, \mathrm{p}_{2}, \ldots, \mathrm{p}_{\mathrm{n}}\right\}$ and the order of probabilities isn't important;

(b) Obtain the cumulative distribution by the function $\mathrm{F}(\mathrm{s})=\sum_{\mathrm{a} \leq \mathrm{s}} \mathrm{p}(\mathrm{a})$;

(c) Consider modified cumulative distribution function $\overline{\mathrm{F}}(\mathrm{s})=\sum_{\mathrm{a}<\mathrm{s}} \mathrm{p}(\mathrm{a})+\frac{1}{2} \mathrm{p}(\mathrm{s})$, where $\overline{\mathrm{F}}(\mathrm{s})$ denotes the sum of probabilities of all symbols less than s plus half the probability of the symbols;

(d) Obtain the length of codeword by the formula $\ell_{\mathrm{i}}(\mathrm{s})=\left\lceil\log \frac{1}{\mathrm{p}(\mathrm{s})}\right\rceil+1$, where $\lceil$. denotes rounding up;

(e) Convert dyadic fraction $\overline{\mathrm{F}}(\mathrm{s})$ to binary form by using Koblitz's trick such that the codeword has $\ell_{\mathrm{i}}(\mathrm{s})$ bits (Cover \& Thomas, 1991).

The concept of optimality of codes There exists a uniquely decodable code whose codeword lengths are given by the sequence $\left\{1_{i}\right\}_{i=1}^{n}$ if Kraft inequality $\sum_{i=1}^{n} 2^{-l_{i}} \leq 1$ holds. Due to Kraft inequality (Cover, 1991), the conditions for optimal codes are as follows:

(a) The average codeword length $\bar{\ell}=\sum_{i=1}^{n} p_{i} \ell_{i}$ of an optimal code for a source $\mathrm{S}$ is greater than or equal to its entropy $H(S)=-\sum_{i=1}^{n} p_{i} \log _{2} p_{i}$; 
(b) The average codeword length $\bar{\ell}$ of an optimal code for a source $\mathrm{S}$ is strictly less than $\mathrm{H}(\mathrm{S})+1$.

For source alphabet $S=\left\{\mathrm{s}_{1}, \mathrm{~s}_{2}, \ldots, \mathrm{s}_{\mathrm{n}}\right\}$ whose probability distribution of occurrences is $\mathrm{P}=\left\{\mathrm{p}_{1}, \mathrm{p}_{2}, \ldots, \mathrm{p}_{\mathrm{n}}\right\}$, the average codeword length is given by $\bar{\ell}$, and entropy of the source alphabet is given by $H(S)$.

Under these conditions, it is required to transmit as well as possible information by using codes consists of fewer bits. So, this problem can be considered as optimization problem which is consist of minimizing $\bar{\ell}=\sum_{\mathrm{i}=1}^{\mathrm{n}} \mathrm{p}_{\mathrm{i}} \ell_{\mathrm{i}}$

subject to constraint $\sum_{i=1}^{n} D^{-l_{i}} \leq 1$, where $\mathrm{D}$ is dimension of codebook, i.e. if the codebook is $\{0,1\}$ then $\mathrm{D}=2$ etc.

This problem is solved by using Langrange Multipliers, and the following result is obtained:

$$
\begin{gathered}
l_{i}^{*}=-\log _{D} p_{i} \\
\bar{l}=\sum_{i=1}^{n} p_{i} l_{i}^{*}=-\sum_{i=1}^{n} p_{i} \log _{D} p_{i}=H_{D}(S) \\
\bar{l}=H_{D}(S) .
\end{gathered}
$$

But it isn't possible to find an interger number for codeword length that satisfies (2.1). For this reason, it is necessary to obtain the entropy lower bound (Cover \& Thomas, 1991; Roman, 1997) satisfying the following inequality:

$$
\bar{l}=\sum_{i=1}^{n} p_{i} l_{i}^{*} \geq H_{D}(S)
$$

Moreover, if $\mathrm{S}$ is a stationary stochastic process,

$$
\bar{l} \rightarrow H(S),
$$

where $\mathrm{H}(\mathrm{S})$ is the entropy rate of the process.

Under the mentioned knowledge, the information per symbol (letter) is given by $I_{\mathrm{inf} / \text { letter }}=\frac{H(S)}{\bar{\ell}}$ and the optimality criteria for codes is considered as $I_{\mathrm{inf} / \text { letter }} \rightarrow 1$ (Венцель, 1969). Moreover, the optimality means that if the text is coded by an optimal coding method, the number of $1 \mathrm{~s}$ and the number of $0 \mathrm{~s}$ are nearly equal in sence of maximum entropy. Hence, the optimal codes means that they transmit nearly maximum information since $1 \mathrm{~s}$ and 0 s aren't always equal probable.

Fano-Huffman Based Statistical Coding Method In this section, a new and improved coding method is proposed, which can be considered as a hybrid method that holds the both advantages of Fano and Huffman coding methods.

It is well known that Fano coding method is a suboptimal procedure for constructing a source code (Rueda \& Oommen, 2004). In this method, the source symbols and their probabilities are sorted in a non-increasing order of the probabilities and then the set of symbols is divided into two subsets such that the sum of the probabilities of occurrences of symbols in each subset are equal or almost equal. The main advantage of this method is the division of the set of symbols. Because, it requires pure computations. Hence, the first goal of the improved coding method is to hold this advantage.

Huffman coding method is a optimal procedure (Cover \& Thomas, 1991). In this method, the source symbols and their probabilities are also sorted in decreasing order and then the two least-probable symbols are merged into a single output whose probability is the sum of the corresponding probabilities. Thus, by this recursive procedure, the optimal Huffman codes are constructed. The advantage of this coding method is that the procedure is from bottom to top. In this way, the short code 
words are attain to the symbols that occur frequently and long code words are attain to the symbols that occur rarely. This advantage of Huffman coding method constitutes the second goal of the improved coding method.

Considering the advantages of these two coding procedure a hybrid coding method is presented. So, the coding method is more easily applicable than the Huffman coding methods and is more optimal than Fano coding method. The codes performed by that coding method are prefix codes and satisfy the sibling property.

The Fano-Huffman based statistical coding method is now proposed in the following form:

(a) Perform the probabilities of symbols in source alphabet in ascending order $\mathrm{p}_{1} \geq \mathrm{p}_{2} \geq \ldots \geq \mathrm{p}_{\mathrm{n}}$

(b) Choose k such that $\left|\sum_{i=1}^{k} p_{i}-\sum_{i=k+1}^{m} p_{i}\right|$ is minimized. This number $\mathrm{k}$ divides the source symbols into two sets of almost equal probability.

(c) Merge the two least-probable letter in each set into a single output whose probability is the sum of the corresponding probabilities;

(d) Go to step (c) if the number of remaining outputs is more than 2 ;

(e) Assign a 0 and a 1 arbitrarily as codewords for the two remaining outputs; (f) Append the current codeword with a 0 and a 1 to obtain the codeword the preceding outputs and repeat step (e) If no output is preceded by another output in a preceding step merge the two least-probable subset into a single output whose probability is the sum of the corresponding probabilities;

(g) Stop if no output is preceded by another output in a preceding step.

Note that, according to step (b) due to size of source alphabet, the set of symbols can be divided into more subsets $\left(2^{\mathrm{n}}, \mathrm{n}=1,2, \ldots\right)$ of equal or almost equal probabilities.

The advantages of the proposed method arise from the comparisons of this method with the other aforesaid coding methods. The applications of this method and comparisons are given in the following section.

Tables, Computational Details and Comparisons

In this section, in order to indicate the advantages of our proposed method, FanoHuffman Based statistical coding method, we compare it with the traditional coding methods. Various binary codes for English, German, Turkish, French, Russian and Spanish symbols are constructed in sense of optimality.

French, German, Spanish and English symbols (letters) are the Latin characters consisting of 26 letters which are given in Table $1 \mathrm{a}$.

The probabilities of French, German and Spanish symbols (letters) were established in 1939 by Fletcher Pratt (Stephens, 2002; Pratt, 1939), the probabilities of English symbols (letters) were established by Nam Phamdo (2001) and they are given in Table $1 \mathrm{~b}$.

Table 1a. French, German, Spanish and English Symbols

$\begin{array}{lllllllllllll}\text { A } & \text { B } & \text { C } & \text { D } & \text { E } & \text { F } & \text { G } & \text { H } & \text { I } & \text { J } & \text { K } & \text { L } & \text { M } \\ \text { a } & \text { B } & \text { c } & \text { d } & \text { e } & \text { F } & \text { g } & \text { h } & \text { i } & \text { j } & \text { k } & \text { l } & \text { m } \\ & & & & & & & & & & & & \\ \text { N } & \text { O } & \text { P } & \text { Q } & \text { R } & \text { S } & \text { T } & \text { U } & \text { V } & \text { W } & \text { X } & \text { Y } & \text { Z } \\ \text { n } & \text { O } & \text { p } & \text { q } & \text { r } & \text { s } & \text { t } & \text { u } & \text { v } & \text { w } & \text { X } & \text { y } & \text { Z }\end{array}$


Table 1b. Probabilities of French, German, Spanish and English Symbols

\begin{tabular}{lllll}
\hline Symbols & English & French & German & Spanish \\
\hline A & 0.065174 & 0.08147 & 0.06506 & 0.12529 \\
B & 0.012425 & 0.00876 & 0.02566 & 0.01420 \\
C & 0.021734 & 0.03063 & 0.02837 & 0.04679 \\
D & 0.034984 & 0.04125 & 0.05414 & 0.05856 \\
E & 0.104144 & 0.17564 & 0.16693 & 0.13676 \\
F & 0.019788 & 0.00959 & 0.02044 & 0.00694 \\
G & 0.015861 & 0.01051 & 0.03647 & 0.01006 \\
H & 0.049289 & 0.00721 & 0.04064 & 0.00704 \\
I & 0.055809 & 0.07559 & 0.07812 & 0.06249 \\
J & 0.000903 & 0.00598 & 0.00191 & 0.00443 \\
K & 0.005053 & 0.00041 & 0.01879 & 0.00004 \\
L & 0.033149 & 0.05783 & 0.02825 & 0.04971 \\
M & 0.020212 & 0.02990 & 0.03005 & 0.03150 \\
N & 0.056451 & 0.07322 & 0.09905 & 0.06712 \\
O & 0.059630 & 0.05289 & 0.02285 & 0.08684 \\
P & 0.013765 & 0.02980 & 0.00944 & 0.02505 \\
Q & 0.000861 & 0.01361 & 0.00055 & 0.00875 \\
R & 0.049756 & 0.06291 & 0.06539 & 0.06873 \\
S & 0.051576 & 0.08013 & 0.06765 & 0.07980 \\
T & 0.072936 & 0.07353 & 0.06742 & 0.04629 \\
U & 0.022513 & 0.05991 & 0.03703 & 0.03934 \\
V & 0.008290 & 0.01557 & 0.01069 & 0.00895 \\
W & 0.017127 & 0.00020 & 0.01396 & 0.00023 \\
X & 0.001369 & 0.00350 & 0.00022 & 0.00221 \\
Y & 0.014598 & 0.00116 & 0.00032 & 0.00895 \\
Z & 0.000784 & 0.00072 & 0.01002 & 0.00523 \\
\# & 0.191818 & - & - & - \\
\hline & & & &
\end{tabular}

Turkish Source Alphabet consists of 29 symbols (letters). The capital and small letters of the Turkish Alphabet are given in Table 2a.

Probabilities of occurrence of Turkish symbols (letters) are given in Table 2b (Shamilov \& Yolacan, 2005; Dalkilic \& Dalkilic, 2002). Considered probabilities have been constituted from a corpus consist of words from many variety of fields, i. e. scientific articles, newspapers, poetics etc., 12.5 million characters in total.

Russian uses Cyrillic alphabet consisting of 32 symbols (letters) which are given in Table 3a. Probabilities of Russian symbols are given in Table 3b., where \# denotes the space symbol (Венцель, 1969; Yaglom \& Yaglom, 1966). 
Table 2a. Turkish Source Alphabet

$\begin{array}{lllllllllllllll}\text { A } & \text { B } & \text { C } & \text { Ç } & \text { D } & \text { E } & \text { F } & \text { G } & \breve{G} & \text { H } & \text { I } & \text { I } & \text { J } & \text { K } & \\ \text { a } & \text { b } & \text { C } & \text { ç } & \text { d } & \text { e } & \text { f } & \text { g } & \breve{g} & \text { h } & 1 & \text { i } & \text { j } & \text { k } & \\ & & & & & & & & & & & & & & \\ \text { L } & M & N & O & O ̈ & \text { P } & \text { R } & \text { S } & \text { Ş } & \text { T } & \text { U } & \text { Ü } & \text { V } & \text { Y } & \text { Z } \\ 1 & \text { m } & \text { N } & \text { o } & \ddot{O} & \text { p } & \text { r } & \text { s } & \text { Ş } & \text { t } & 1 & \text { ü } & \text { v } & \text { y } & \text { Z }\end{array}$

Table 2b. Probabilities of Turkish Symbols

\begin{tabular}{llllll}
\hline Letter & Frequency & Letter & Frequency & Letter & Frequency \\
\hline $\mathrm{A}$ & 0.1026 & $\mathrm{I}$ & 0.0444 & $\mathrm{R}$ & 0.0604 \\
$\mathrm{~B}$ & 0.0237 & I & 0.0723 & $\mathrm{~S}$ & 0.0264 \\
$\mathrm{C}$ & 0.0084 & $\mathrm{~J}$ & 0.0003 & $\mathrm{~S}$ & 0.0157 \\
$\mathrm{C}$ & 0.0102 & $\mathrm{~K}$ & 0.0407 & $\mathrm{~T}$ & 0.0287 \\
$\mathrm{D}$ & 0.0400 & $\mathrm{~L}$ & 0.0530 & $\mathrm{U}$ & 0.0284 \\
$\mathrm{E}$ & 0.0782 & $\mathrm{M}$ & 0.0320 & Ü & 0.0171 \\
$\mathrm{~F}$ & 0.0038 & $\mathrm{~N}$ & 0.0633 & $\mathrm{~V}$ & 0.0087 \\
$\mathrm{G}$ & 0.0114 & $\mathrm{O}$ & 0.0214 & $\mathrm{Y}$ & 0.0295 \\
$\breve{G}$ & 0.0092 & Ö & 0.0074 & $\mathrm{Z}$ & 0.0130 \\
$\mathrm{H}$ & 0.0096 & $\mathrm{P}$ & 0.0073 & $\#$ & 0.1329 \\
\hline
\end{tabular}

Table 3a. Russian Symbols (Cyrillic alphabet)
$\begin{array}{lllllllllllllll}\text { А } & Б & \text { В } & \text { Г } & \text { Д } & \text { Е } & Ж & 3 & И & \breve{~} & \text { К } & Л & \text { M } & \text { Н } & \text { О }\end{array}$

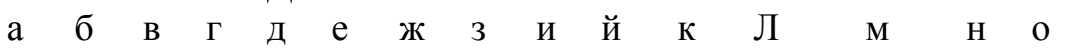

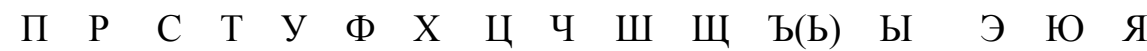

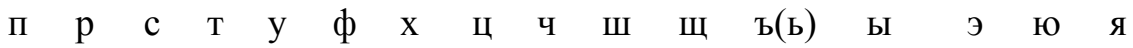

Table 3b. Probabilities of Russian Symbols

\begin{tabular}{|c|c|c|c|}
\hline Symbols & Probabilities & Symbols & Probabilities \\
\hline A & 0.064 & $\mathrm{P}$ & 0.041 \\
\hline Б & 0.015 & $\mathrm{C}$ & 0.047 \\
\hline B & 0.039 & $\mathrm{~T}$ & 0.056 \\
\hline$\Gamma$ & 0.014 & $\mathrm{y}$ & 0.021 \\
\hline Д & 0.026 & $\Phi$ & 0.002 \\
\hline $\mathrm{E}$ & 0.074 & $X$ & 0.009 \\
\hline Ж & 0.008 & Ц & 0.004 \\
\hline 3 & 0.015 & Ч & 0.013 \\
\hline И & 0.064 & Ш & 0.006 \\
\hline Й & 0.010 & Щ & 0.003 \\
\hline K & 0.029 & $\mathrm{~b}(\mathrm{~b})$ & 0.015 \\
\hline Л & 0.036 & Ы & 0.016 \\
\hline M & 0.026 & Э & 0.003 \\
\hline $\mathrm{H}$ & 0.056 & Ю & 0.007 \\
\hline $\mathrm{O}$ & 0.095 & Я & 0.019 \\
\hline$\Pi$ & 0.024 & \# & 0.145 \\
\hline
\end{tabular}


In order to construct binary codes for English, German, Turkish, French, Russian and Spanish, the classical coding methods are applied to considered source alphabets. Consequently, the constructed binary codes are given respectively in Tables 4-9. Moreover, Fano-Huffman Based statistical coding method is also applied to considered languages. Binary Codes constructed by Fano-Huffman based statistical coding are given in Table 10.

Table 4 Binary Codes for Probability Distrubution of English Symbols

\begin{tabular}{|c|c|c|c|c|c|c|c|c|}
\hline $\begin{array}{l}\text { English } \\
\text { Alphabet }\end{array}$ & No & $\begin{array}{l}\text { Ordinary } \\
\text { Codes }\end{array}$ & $\begin{array}{l}\text { S-F-E } \\
\text { Codes }\end{array}$ & $\begin{array}{l}\text { Ordered } \\
\text { Alphabet }\end{array}$ & $\begin{array}{l}\text { Shannon } \\
\text { Codes }\end{array}$ & Fano Codes & $\begin{array}{l}\text { Enhanced } \\
\text { Fano } \\
\text { Codes }\end{array}$ & $\begin{array}{l}\text { Huffman } \\
\text { Codes }\end{array}$ \\
\hline A & 0 & 00000 & 00001 & \# & 000 & 000 & 000 & 001 \\
\hline B & 1 & 00001 & 00010010 & $\mathrm{E}$ & 0011 & 001 & 001 & 100 \\
\hline $\mathrm{C}$ & 2 & 00010 & 0001011 & $\mathrm{~T}$ & 0100 & 010 & 010 & 0101 \\
\hline D & 3 & 00011 & 000111 & A & 0101 & 0110 & 0110 & 0011 \\
\hline $\mathrm{E}$ & 4 & 00100 & 00101 & $\mathrm{O}$ & 01101 & 0111 & 0111 & 0111 \\
\hline $\mathrm{F}$ & 5 & 00101 & 0011111 & $\mathrm{~N}$ & 01111 & 1000 & 1000 & 0000 \\
\hline G & 6 & 00110 & 0100010 & I & 10001 & 1001 & 1001 & 1000 \\
\hline $\mathrm{H}$ & 7 & 00111 & 010011 & $\mathrm{~S}$ & 10011 & 1010 & 1010 & 0010 \\
\hline I & 8 & 01000 & 010110 & $\mathrm{R}$ & 10100 & 10110 & 10110 & 1010 \\
\hline $\mathrm{J}$ & 9 & 01001 & 011000010011 & $\mathrm{H}$ & 10110 & 10111 & 10111 & 0110 \\
\hline K & 10 & 01010 & 011000011 & $\mathrm{D}$ & 11000 & 11000 & 11000 & 01101 \\
\hline $\mathrm{L}$ & 11 & 01011 & 011001 & $\mathrm{~L}$ & 11001 & 11001 & 11001 & 01011 \\
\hline M & 12 & 01100 & 0110110 & $\mathrm{U}$ & 110100 & 11010 & 11010 & 01110 \\
\hline $\mathrm{N}$ & 13 & 01101 & 011101 & $\mathrm{C}$ & 110110 & 110111 & 11100 & 11110 \\
\hline $\mathrm{O}$ & 14 & 01110 & 100001 & M & 110111 & 110110 & 110110 & 011101 \\
\hline $\mathrm{P}$ & 15 & 01111 & 10001111 & $\mathrm{~F}$ & 111000 & 11100 & 110111 & 111101 \\
\hline Q & 16 & 10000 & 100100011001 & W & 111001 & 111010 & 111010 & 011011 \\
\hline $\mathrm{R}$ & 17 & 10001 & 100110 & $\mathrm{G}$ & 111011 & 111011 & 111011 & 001111 \\
\hline $\mathrm{S}$ & 18 & 10010 & 101001 & $\mathrm{Y}$ & 1111000 & 111100 & 111100 & 101111 \\
\hline $\mathrm{T}$ & 19 & 10011 & 10110 & $\mathrm{P}$ & 1111010 & 111101 & 111101 & 011111 \\
\hline $\mathrm{U}$ & 20 & 10100 & 1100000 & B & 1111011 & 111110 & 111110 & 111111 \\
\hline $\mathrm{V}$ & 21 & 10101 & 11000101 & $\mathrm{~V}$ & 1111101 & 1111110 & 1111110 & 0111011 \\
\hline W & 22 & 10110 & 1100100 & $\mathrm{~K}$ & 11111100 & 11111110 & 11111110 & 01111011 \\
\hline $\mathrm{X}$ & 23 & 10111 & 110001010110 & $\mathrm{X}$ & 1111111000 & 111111110 & 111111110 & 011111011 \\
\hline Y & 24 & 11000 & 11001100 & $\mathrm{~J}$ & 11111110100 & 1111111110 & 1111111110 & 1011111011 \\
\hline Z & 25 & 11001 & 110011101100 & $\mathrm{Q}$ & 11111110110 & 11111111110 & 11111111110 & 0111111011 \\
\hline \# & 26 & 11010 & 1110 & Z & 11111110111 & 11111111111 & 11111111111 & 1111111011 \\
\hline
\end{tabular}


Table 5. Binary Codes for Probability Distrubution of German Symbols

\begin{tabular}{lllllllll}
\hline $\begin{array}{l}\text { German } \\
\text { Alphabet }\end{array}$ & No & $\begin{array}{l}\text { Ordinary } \\
\text { Codes }\end{array}$ & $\begin{array}{l}\text { S-F-E } \\
\text { Codes }\end{array}$ & $\begin{array}{l}\text { Ordered } \\
\text { Alphabet }\end{array}$ & $\begin{array}{l}\text { Shannon } \\
\text { Codes }\end{array}$ & Fano Codes & $\begin{array}{l}\text { Enhanced } \\
\text { Fano } \\
\text { Codes }\end{array}$ & $\begin{array}{l}\text { Huffman } \\
\text { Codes }\end{array}$ \\
\hline A & 0 & 00000 & 00001 & E & 000 & 000 & 000 & 000 \\
B & 1 & 00001 & 0001001 & N & 0010 & 001 & 001 & 001 \\
C & 2 & 00010 & 0001101 & I & 0100 & 010 & 010 & 0100 \\
D & 3 & 00011 & 001001 & S & 0101 & 0110 & 0110 & 0010 \\
E & 4 & 00100 & 0100 & T & 0110 & 0111 & 0111 & 1010 \\
F & 5 & 00101 & 0101100 & R & 0111 & 1000 & 1000 & 0110 \\
G & 6 & 00110 & 011000 & A & 1000 & 1001 & 1001 & 0011 \\
H & 7 & 00111 & 011010 & D & 10011 & 1010 & 1010 & 0111 \\
I & 8 & 01000 & 01111 & H & 10101 & 10110 & 10110 & 0101 \\
J & 9 & 01001 & 10000100010 & U & 10110 & 10111 & 10111 & 01100 \\
K & 10 & 01010 & 1000011 & G & 10111 & 11000 & 11000 & 01110 \\
L & 11 & 01011 & 1000110 & M & 110001 & 11001 & 11001 & 11110 \\
M & 12 & 01100 & 1001010 & $\mathrm{C}$ & 110011 & 11010 & 11010 & 01011 \\
N & 13 & 01101 & 10100 & L & 110101 & 11011 & 11011 & 11011 \\
O & 14 & 01110 & 1011010 & B & 110111 & 11100 & 11100 & 01111 \\
P & 15 & 01111 & 10111000 & O & 111000 & 111010 & 111010 & 01101 \\
Q & 16 & 10000 & 101110011111 & F & 111010 & 111011 & 111011 & 011100 \\
R & 17 & 10001 & 11000 & K & 111011 & 111100 & 111100 & 111100 \\
S & 18 & 10010 & 11010 & W & 1111001 & 111101 & 111101 & 011111 \\
T & 19 & 10011 & 11100 & V & 1111011 & 1111100 & 1111100 & 011101 \\
U & 20 & 10100 & 111100 & Z & 1111101 & 1111101 & 1111101 & 111101 \\
V & 21 & 10101 & 11111000 & P & 1111110 & 1111110 & 1111110 & 0111111 \\
W & 22 & 10110 & 11111011 & J & 1111111100 & 11111110 & 11111110 & 01111111 \\
X & 23 & 10111 & 11111101001011 & Q & 11111111100 & 111111110 & 111111110 & 011111111 \\
Y & 24 & 11000 & 1111110100111 & Y & 111111111011 & 1111111110 & 1111111110 & 0111111111 \\
Z & 25 & 11001 & 11111110 & X & 1111111111001 & 1111111111 & 1111111111 & 1111111111 \\
\hline
\end{tabular}

Table 6. Binary Codes for Probability Distrubution of Turkish Symbols

\begin{tabular}{|c|c|c|c|c|c|c|c|c|}
\hline $\begin{array}{l}\text { Turkish } \\
\text { Alphabet }\end{array}$ & No & $\begin{array}{l}\text { Ordinary } \\
\text { Codes }\end{array}$ & $\begin{array}{l}\text { S-F-E } \\
\text { Codes }\end{array}$ & $\begin{array}{l}\text { Ordered } \\
\text { Alphabet }\end{array}$ & $\begin{array}{l}\text { Shannon } \\
\text { Codes }\end{array}$ & Fano Codes & $\begin{array}{l}\text { Enhanced } \\
\text { Fano } \\
\text { Codes }\end{array}$ & $\begin{array}{l}\text { Huffman } \\
\text { Codes }\end{array}$ \\
\hline A & 0 & 00000 & 00001 & \# & 000 & 000 & 000 & 001 \\
\hline B & 1 & 00001 & 0001110 & A & 0010 & 001 & 001 & 000 \\
\hline $\mathrm{C}$ & 2 & 00010 & 00100001 & E & 0011 & 0100 & 0100 & 0011 \\
\hline Ç & 3 & 00011 & 00100011 & İ & 0101 & 0101 & 0101 & 0111 \\
\hline $\mathrm{D}$ & 4 & 00100 & 001010 & $\mathrm{~N}$ & 0110 & 0110 & 0110 & 0101 \\
\hline $\mathrm{E}$ & 5 & 00101 & 00111 & $\mathrm{R}$ & 01110 & 0111 & 0111 & 0010 \\
\hline $\mathrm{F}$ & 6 & 00110 & 0100001111 & $\mathrm{~L}$ & 10000 & 1000 & 1000 & 0110 \\
\hline G & 7 & 00111 & 01000101 & I & 10010 & 1001 & 1001 & 0100 \\
\hline$\breve{G}$ & 8 & 01000 & 01001000 & $\mathrm{~K}$ & 10011 & 10100 & 10100 & 01011 \\
\hline $\mathrm{H}$ & 9 & 01001 & 01001010 & $\mathrm{D}$ & 10100 & 10101 & 10101 & 01111 \\
\hline I & 10 & 01010 & 010100 & M & 10110 & 10110 & 10110 & 01101 \\
\hline İ & 11 & 01011 & 01100 & $\mathrm{Y}$ & 101110 & 10111 & 10111 & 01010 \\
\hline $\mathrm{J}$ & 12 & 01100 & 0110100111111 & $\mathrm{~T}$ & 101111 & 11000 & 11000 & 11010 \\
\hline K & 13 & 01101 & 011011 & U & 110001 & 11001 & 11001 & 01110 \\
\hline $\mathrm{L}$ & 14 & 01110 & 011110 & $\mathrm{~S}$ & 110011 & 11010 & 11010 & 01100 \\
\hline M & 15 & 01111 & 100001 & B & 110101 & 110110 & 110110 & 11100 \\
\hline $\mathrm{N}$ & 16 & 10000 & 10010 & $\mathrm{O}$ & 110110 & 110111 & 110111 & 011011 \\
\hline $\mathrm{O}$ & 17 & 10001 & 1001110 & Ü & 111000 & 111000 & 111000 & 011101 \\
\hline Ö & 18 & 10010 & 101000001 & Ş & 111001 & 111001 & 111001 & 011110 \\
\hline $\mathrm{P}$ & 19 & 10011 & 101000101 & $\mathrm{Z}$ & 1110100 & 111010 & 111010 & 111110 \\
\hline $\mathrm{R}$ & 20 & 10100 & 101010 & G & 1110110 & 111011 & 111011 & 0111011 \\
\hline $\mathrm{S}$ & 21 & 10101 & 1011011 & $\mathrm{C}$ & 1110111 & 1111000 & 1111000 & 0011111 \\
\hline Ș & 22 & 10110 & 1011101 & $\mathrm{H}$ & 1111000 & 1111001 & 1111001 & 1011111 \\
\hline $\mathrm{T}$ & 23 & 10111 & 1100000 & $\breve{G}$ & 1111010 & 1111010 & 1111010 & 0111111 \\
\hline $\mathrm{U}$ & 24 & 11000 & 1100100 & V & 1111011 & 1111011 & 1111011 & 1111111 \\
\hline Ü & 25 & 11001 & 1100111 & $\mathrm{C}$ & 1111100 & 1111100 & 1111100 & 0111101 \\
\hline V & 26 & 11010 & 11010001 & Ö & 11111011 & 1111101 & 1111101 & 1111101 \\
\hline $\mathrm{Y}$ & 27 & 11011 & 1101011 & $\mathrm{P}$ & 11111101 & 1111110 & 1111110 & 01111011 \\
\hline Z & 28 & 11100 & 11011100 & $\mathrm{~F}$ & 111111101 & 11111110 & 11111110 & 011111011 \\
\hline \# & 29 & 11101 & 1110 & $\mathrm{~J}$ & 111111111110 & 11111111 & 11111111 & 111111011 \\
\hline
\end{tabular}


Table 7. Binary Codes for Probability Distrubution of French Symbols

\begin{tabular}{|c|c|c|c|c|c|c|c|c|}
\hline $\begin{array}{l}\text { French } \\
\text { Alphabet }\end{array}$ & No & $\begin{array}{l}\text { Ordinary } \\
\text { Codes }\end{array}$ & $\begin{array}{l}\text { S-F-E } \\
\text { Codes }\end{array}$ & $\begin{array}{l}\text { Ordered } \\
\text { Alphabet }\end{array}$ & $\begin{array}{l}\text { Shannon } \\
\text { Codes }\end{array}$ & Fano Codes & $\begin{array}{l}\text { Enhanced } \\
\text { Fano } \\
\text { Codes }\end{array}$ & $\begin{array}{l}\text { Huffman } \\
\text { Codes }\end{array}$ \\
\hline $\mathrm{A}$ & 0 & 00000 & 00001 & $\mathrm{E}$ & 000 & 000 & 000 & 00 \\
\hline B & 1 & 00001 & 00010101 & A & 0010 & 001 & 001 & 0101 \\
\hline $\mathrm{C}$ & 2 & 00010 & 0001101 & $\mathrm{~S}$ & 0100 & 010 & 010 & 0001 \\
\hline D & 3 & 00011 & 001001 & I & 0101 & 0110 & 0110 & 1001 \\
\hline E & 4 & 00100 & 0011 & $\mathrm{~T}$ & 0110 & 0111 & 0111 & 0011 \\
\hline $\mathrm{F}$ & 5 & 00101 & 01010111 & $\mathrm{~N}$ & 0111 & 1000 & 1000 & 1011 \\
\hline G & 6 & 00110 & 01011010 & $\mathrm{R}$ & 1000 & 1001 & 1001 & 0111 \\
\hline $\mathrm{H}$ & 7 & 00111 & 010111001 & $\mathrm{U}$ & 10011 & 1010 & 1010 & 0110 \\
\hline I & 8 & 01000 & 01100 & $\mathrm{~L}$ & 10101 & 1011 & 1011 & 0010 \\
\hline J & 9 & 01001 & 011100011 & $\mathrm{O}$ & 10111 & 1100 & 1100 & 1010 \\
\hline $\mathrm{K}$ & 10 & 01010 & 0111001001100 & $\mathrm{D}$ & 11001 & 11010 & 11010 & 01101 \\
\hline $\mathrm{L}$ & 11 & 01011 & 011110 & $\mathrm{C}$ & 110101 & 11011 & 11011 & 01111 \\
\hline M & 12 & 01100 & 1000010 & M & 110111 & 11100 & 11100 & 01110 \\
\hline $\mathrm{N}$ & 13 & 01101 & 10010 & $\mathrm{P}$ & 111001 & 111010 & 111010 & 11110 \\
\hline $\mathrm{O}$ & 14 & 01110 & 101000 & $\mathrm{~V}$ & 1110110 & 111011 & 111011 & 011111 \\
\hline $\mathrm{P}$ & 15 & 01111 & 1010110 & Q & 1111000 & 111100 & 111100 & 0111101 \\
\hline Q & 16 & 10000 & 10110010 & $\mathrm{G}$ & 1111010 & 1111010 & 1111010 & 0011101 \\
\hline $\mathrm{R}$ & 17 & 10001 & 10111 & $\mathrm{~F}$ & 1111011 & 1111011 & 1111011 & 1011101 \\
\hline $\mathrm{S}$ & 18 & 10010 & 11001 & B & 1111100 & 1111100 & 1111100 & 0111111 \\
\hline $\mathrm{T}$ & 19 & 10011 & 11100 & $\mathrm{H}$ & 11111011 & 1111101 & 1111101 & 1111111 \\
\hline $\mathrm{U}$ & 20 & 10100 & 111100 & $\mathrm{~J}$ & 11111101 & 1111110 & 1111110 & 01111101 \\
\hline $\mathrm{V}$ & 21 & 10101 & 11111101 & $\mathrm{X}$ & 111111101 & 11111110 & 11111110 & 011111101 \\
\hline W & 22 & 10110 & 11111111000101 & $\mathrm{Y}$ & 1111111111 & 111111110 & 111111110 & 0111111101 \\
\hline $\mathrm{X}$ & 23 & 10111 & 1111111110 & Z & 00000000001 & 1111111110 & 1111111110 & 01111111101 \\
\hline $\mathrm{Y}$ & 24 & 11000 & 00000000001 & $\mathrm{~K}$ & 000000000101 & 11111111110 & 11111111110 & 011111111101 \\
\hline Z & 25 & 11001 & 000000000110 & W & 0000000001110 & 11111111111 & 11111111111 & 111111111101 \\
\hline
\end{tabular}

Table 8. Binary Codes for Probability Distrubution of Russian Symbols

\begin{tabular}{|c|c|c|c|c|c|c|c|c|}
\hline $\begin{array}{l}\text { Russian } \\
\text { Alphabet }\end{array}$ & No & $\begin{array}{l}\text { Ordinary } \\
\text { Codes }\end{array}$ & $\begin{array}{l}\text { S-F-E } \\
\text { Codes }\end{array}$ & $\begin{array}{l}\text { Ordered } \\
\text { Alphabet }\end{array}$ & $\begin{array}{l}\text { Shannon } \\
\text { Codes }\end{array}$ & Fano Codes & $\begin{array}{l}\text { Enhanced } \\
\text { Fano } \\
\text { Codes }\end{array}$ & $\begin{array}{l}\text { Huffman } \\
\text { Codes }\end{array}$ \\
\hline A & 0 & 00000 & 00010 & \# & 000 & 000 & 000 & 000 \\
\hline Б & 1 & 00001 & 00010010 & $\mathrm{O}$ & 0010 & 001 & 001 & 001 \\
\hline B & 2 & 00010 & 000110 & $\mathrm{E}$ & 0011 & 0100 & 0100 & 011 \\
\hline$\Gamma$ & 3 & 00011 & 00100000 & $\mathrm{~A}$ & 0101 & 0101 & 0101 & 0010 \\
\hline Д & 4 & 00100 & 0010001 & И & 0110 & 0110 & 0110 & 1010 \\
\hline $\mathrm{E}$ & 5 & 00101 & 00110 & $\mathrm{~T}$ & 01110 & 0111 & 0111 & 0111 \\
\hline Ж & 6 & 00110 & 00111100 & $\mathrm{H}$ & 01111 & 1000 & 1000 & 0011 \\
\hline 3 & 7 & 00111 & 00111111 & $\mathrm{C}$ & 10001 & 1001 & 1001 & 0101 \\
\hline И & 8 & 01000 & 01001 & $\mathrm{P}$ & 10011 & 10100 & 10100 & 00100 \\
\hline Й & 9 & 01001 & 01010010 & B & 10100 & 10101 & 10101 & 01100 \\
\hline K & 10 & 01010 & 0101011 & Л & 10101 & 10110 & 10110 & 01110 \\
\hline Л & 11 & 01011 & 011000 & K & 101101 & 10111 & 10111 & 01011 \\
\hline M & 12 & 01100 & 0110100 & M & 101111 & 11000 & 11000 & 01101 \\
\hline $\mathrm{H}$ & 13 & 01101 & 011100 & Д & 110001 & 110010 & 11010 & 11101 \\
\hline $\mathrm{O}$ & 14 & 01110 & 10000 & $\bar{\Pi}$ & 110011 & 110011 & 110010 & 010100 \\
\hline П & 15 & 01111 & 1001010 & $\mathrm{y}$ & 110100 & 11010 & 110011 & 110100 \\
\hline $\mathrm{P}$ & 16 & 10000 & 100111 & Я & 110101 & 110110 & 110110 & 011100 \\
\hline $\mathrm{C}$ & 17 & 10001 & 101010 & Ы & 110111 & 110111 & 110111 & 011110 \\
\hline $\mathrm{T}$ & 18 & 10010 & 101101 & 3 & 1110000 & 111000 & 111000 & 001111 \\
\hline $\mathrm{y}$ & 19 & 10011 & 1011111 & $\mathrm{~b}(\mathrm{~b})$ & 1110010 & 111001 & 111001 & 101111 \\
\hline$\Phi$ & 20 & 10100 & 1100001011 & Б & 1110100 & 111010 & 111010 & 011111 \\
\hline $\mathrm{X}$ & 21 & 10101 & 11000100 & $\Gamma$ & 1110110 & 111011 & 111011 & 011011 \\
\hline Ц & 22 & 10110 & 110001011 & Ч & 1110111 & 111100 & 111100 & 111011 \\
\hline Ч & 23 & 10111 & 11001000 & Й & 1111001 & 1111010 & 1111010 & 0111100 \\
\hline Ш & 24 & 11000 & 110010100 & $\mathrm{X}$ & 1111010 & 1111011 & 1111011 & 0111110 \\
\hline щ & 25 & 11001 & 1100101110 & Ж & 1111100 & 1111100 & 1111100 & 1111110 \\
\hline $\mathrm{b}(\mathrm{b})$ & 26 & 11010 & 11001101 & Ю & 11111010 & 1111101 & 1111101 & 0111111 \\
\hline Ы & 27 & 11011 & 1101000 & Ш & 11111011 & 11111100 & 11111100 & 01111100 \\
\hline Э & 28 & 11100 & 1101010001 & Ц & 11111101 & 11111101 & 11111101 & 11111100 \\
\hline Ю & 29 & 11101 & 110101011 & Щ & 111111100 & 11111110 & 11111110 & 01111111 \\
\hline Я & 30 & 11110 & 1101100 & $\ni$ & 111111110 & 111111110 & 111111110 & 011111111 \\
\hline \# & 31 & 11111 & 1110 & $\Phi$ & 111111111 & 111111111 & 111111111 & 111111111 \\
\hline
\end{tabular}


Table 9. Binary Codes for Probability Distrubution of Spanish Symbols

\begin{tabular}{|c|c|c|c|c|c|c|c|c|}
\hline $\begin{array}{l}\text { Spanish } \\
\text { Symbols }\end{array}$ & No & $\begin{array}{l}\text { Ordinary } \\
\text { Codes }\end{array}$ & $\begin{array}{l}\text { S-F-E } \\
\text { Codes }\end{array}$ & $\begin{array}{l}\text { Ordered } \\
\text { Alphabet }\end{array}$ & $\begin{array}{l}\text { Shannon } \\
\text { Codes }\end{array}$ & Fano Codes & $\begin{array}{l}\text { Enhanced } \\
\text { Fano } \\
\text { Codes }\end{array}$ & $\begin{array}{l}\text { Huffman } \\
\text { Codes }\end{array}$ \\
\hline A & 0 & 00000 & 0001 & E & 000 & 000 & 000 & 000 \\
\hline B & 1 & 00001 & 00100001 & A & 001 & 001 & 001 & 001 \\
\hline $\mathrm{C}$ & 2 & 00010 & 001010 & $\mathrm{O}$ & 0100 & 010 & 010 & 0010 \\
\hline D & 3 & 00011 & 001101 & $\mathrm{~S}$ & 0101 & 0110 & 0110 & 1010 \\
\hline E & 4 & 00100 & 0101 & $\mathrm{R}$ & 0110 & 0111 & 0111 & 0110 \\
\hline $\mathrm{F}$ & 5 & 00101 & 011000101 & $\mathrm{~N}$ & 0111 & 1000 & 1000 & 0100 \\
\hline G & 6 & 00110 & 01100100 & I & 10010 & 1001 & 1001 & 1100 \\
\hline $\mathrm{H}$ & 7 & 00111 & 011001101 & $\mathrm{D}$ & 10100 & 1010 & 1010 & 0101 \\
\hline I & 8 & 01000 & 011011 & $\mathrm{~L}$ & 10101 & 1011 & 1011 & 0011 \\
\hline $\mathrm{J}$ & 9 & 01001 & 011110000 & $\mathrm{C}$ & 10111 & 11000 & 11000 & 1011 \\
\hline $\mathrm{K}$ & 10 & 01010 & 0111100011111011 & $\mathrm{~T}$ & 11001 & 11001 & 11001 & 0111 \\
\hline $\mathrm{L}$ & 11 & 01011 & 011111 & $\mathrm{U}$ & 11010 & 11010 & 11010 & 01110 \\
\hline M & 12 & 01100 & 100010 & M & 11011 & 11011 & 11011 & 01101 \\
\hline $\mathrm{N}$ & 13 & 01101 & 10010 & $\mathrm{P}$ & 111001 & 11100 & 11100 & 01111 \\
\hline $\mathrm{O}$ & 14 & 01110 & 10101 & B & 1110110 & 111010 & 111010 & 011110 \\
\hline $\mathrm{P}$ & 15 & 01111 & 1011100 & $\mathrm{G}$ & 1111000 & 111011 & 111011 & 011111 \\
\hline Q & 16 & 10000 & 10111100 & $\mathrm{Y}$ & 1111001 & 111100 & 111100 & 111111 \\
\hline $\mathrm{R}$ & 17 & 10001 & 11000 & $\mathrm{~V}$ & 1111010 & 1111010 & 1111010 & 0111110 \\
\hline $\mathrm{S}$ & 18 & 10010 & 11011 & $\mathrm{Q}$ & 1111011 & 1111011 & 1111011 & 1111110 \\
\hline $\mathrm{T}$ & 19 & 10011 & 111010 & $\mathrm{H}$ & 11111001 & 1111100 & 1111100 & 0011101 \\
\hline $\mathrm{U}$ & 20 & 10100 & 111101 & $\mathrm{~F}$ & 11111011 & 1111101 & 1111101 & 1011101 \\
\hline $\mathrm{V}$ & 21 & 10101 & 11111010 & $\mathrm{Z}$ & 11111101 & 1111110 & 1111110 & 0111101 \\
\hline W & 22 & 10110 & 11111100000110 & $\mathrm{~J}$ & 11111110 & 11111110 & 11111110 & 01111101 \\
\hline $\mathrm{X}$ & 23 & 10111 & 1111110001 & $\mathrm{X}$ & 111111111 & 111111110 & 111111110 & 011111101 \\
\hline $\mathrm{Y}$ & 24 & 11000 & 11111101 & W & 0000000001000 & 1111111110 & 1111111110 & 0111111101 \\
\hline Z & 25 & 11001 & 111111111 & $\mathrm{~K}$ & 000000000101001 & 1111111111 & 1111111111 & 1111111101 \\
\hline
\end{tabular}

Table 10. Binary Codes Constructed by Fano-Huffman Based Statistical Coding Method

\begin{tabular}{|c|c|c|c|c|c|c|c|c|}
\hline $\begin{array}{l}\text { Turkish } \\
\text { Alphabet }\end{array}$ & $\begin{array}{l}\text { Fano- } \\
\text { Huffman } \\
\text { based } \\
\text { Codes for } \\
\text { Turkish } \\
\text { symbols }\end{array}$ & $\begin{array}{l}\text { Russian } \\
\text { Alphabet }\end{array}$ & $\begin{array}{l}\text { Fano- } \\
\text { Huffman } \\
\text { based Codes } \\
\text { for Russian } \\
\text { symbols }\end{array}$ & $\begin{array}{l}\text { English, } \\
\text { French, } \\
\text { German, } \\
\text { Spanish } \\
\text { Alphabet }\end{array}$ & $\begin{array}{l}\text { Fano- } \\
\text { Huffman } \\
\text { based } \\
\text { Codes for } \\
\text { English } \\
\text { symbols }\end{array}$ & $\begin{array}{l}\text { Fano- } \\
\text { Huffman } \\
\text { based } \\
\text { Codes for } \\
\text { French } \\
\text { symbols }\end{array}$ & $\begin{array}{l}\text { Fano-Huffman } \\
\text { based } \\
\text { Codes for } \\
\text { German } \\
\text { symbols }\end{array}$ & $\begin{array}{l}\text { Fano-Huffman } \\
\text { based } \\
\text { Codes for } \\
\text { Spanish } \\
\text { symbols }\end{array}$ \\
\hline $\mathrm{A}$ & 110 & $\mathrm{~A}$ & 1101 & $\mathrm{~A}$ & 0101 & 0001 & 0010 & 011 \\
\hline B & 10111 & Б & 011100 & B & 111100 & 0001100 & 11110 & 111000 \\
\hline $\mathrm{C}$ & 0100101 & B & 11000 & $\mathrm{C}$ & 110000 & 11100 & 01010 & 1110 \\
\hline Ç & 0011001 & $\Gamma$ & 011010 & D & 11000 & 10000 & 0110 & 0010 \\
\hline $\mathrm{D}$ & 01001 & Д & 10110 & E & 0001 & 11 & 001 & 101 \\
\hline $\mathrm{E}$ & 0000 & $\mathrm{E}$ & 0101 & $\mathrm{~F}$ & 101000 & 1100000 & 001000 & 1011010 \\
\hline $\mathrm{F}$ & 010000001 & Ж & 1010100 & G & 001100 & 0100000 & 00100 & 0110000 \\
\hline G & 1000001 & 3 & 001100 & $\mathrm{H}$ & 00000 & 1001100 & 10000 & 0011010 \\
\hline$\breve{G}$ & 0111001 & И & 0011 & I & 1010 & 0101 & 111 & 1100 \\
\hline $\mathrm{H}$ & 1011001 & $\breve{И}$ & 1100000 & $\mathrm{~J}$ & 1010000100 & 11000000 & 01101110 & 00111010 \\
\hline I & 1111 & K & 01010 & $\mathrm{~K}$ & 00000100 & 010101000000 & 101000 & 1110111010 \\
\hline$\dot{I}$ & 1000 & Л & 00100 & $\mathrm{~L}$ & 10100 & 0110 & 11010 & 0110 \\
\hline $\mathrm{J}$ & 110000001 & M & 00110 & M & 001000 & 01010 & 10100 & 01010 \\
\hline K & 10001 & $\mathrm{H}$ & 0010 & $\mathrm{~N}$ & 0010 & 1000 & 011 & 0100 \\
\hline $\mathrm{L}$ & 1011 & $\mathrm{O}$ & 111 & $\mathrm{O}$ & 1101 & 1110 & 000000 & 111 \\
\hline M & 10101 & $\Pi$ & 000000 & $\mathrm{P}$ & 011100 & 11010 & 0101110 & 010000 \\
\hline $\mathrm{N}$ & 0010 & $\mathrm{P}$ & 10000 & Q & 0110000100 & 0000000 & 011101110 & 1011000 \\
\hline $\mathrm{O}$ & 100001 & $\mathrm{C}$ & 1110 & $\mathrm{R}$ & 1110 & 0100 & 1100 & 1001 \\
\hline Ö & 1100101 & $\mathrm{~T}$ & 1011 & S & 0110 & 1001 & 0101 & 0001 \\
\hline $\mathrm{P}$ & 00000001 & $\mathrm{y}$ & 001000 & $\mathrm{~T}$ & 1001 & 1101 & 1101 & 00000 \\
\hline $\mathrm{R}$ & 1010 & $\Phi$ & 110100000 & $\mathrm{U}$ & 010000 & 0010 & 11000 & 01000 \\
\hline S & 00111 & $X$ & 0010100 & $\mathrm{~V}$ & 1000100 & 101100 & 0100000 & 0011000 \\
\hline Ş & 011101 & Ц & 01111100 & W & 100100 & 110101000000 & 001110 & 0110111010 \\
\hline $\mathrm{T}$ & 00011 & $\mathrm{\Psi}$ & 111010 & $\mathrm{X}$ & 0010000100 & 001000000 & 1111101110 & 010111010 \\
\hline $\mathrm{U}$ & 10011 & Ш & 00100000 & Y & 101100 & 1101000000 & 0111101110 & 1110000 \\
\hline$\ddot{U}$ & 000101 & щ & 11111100 & Z & 1110000100 & 00101000000 & 1100000 & 1111010 \\
\hline V & 1111001 & $\mathrm{~b}(\mathrm{~b})$ & 101100 & $\#$ & 11 & & & \\
\hline Y & 01101 & Ы & 110100 & & & & & \\
\hline Z & 111101 & $\ni$ & 010100000 & & & & & \\
\hline \multirow[t]{3}{*}{ \# } & 100 & Ю & 0111100 & & & & & \\
\hline & & Я & 101000 & & & & & \\
\hline & & \# & 001 & & & & & \\
\hline
\end{tabular}


In order to determine the information per letter for considered alphabets due to the mentioned coding methods, the following stages are presented:

1) The entropy of each mentioned languages $H(S)$ is calculated.

2) The codeword length of each codes shown in Tables 4-10 is obtained by counting the bits of the code words and thus average codeword length $\bar{\ell}$ is computed for each coding methods.

3) The information per letter $\mathrm{I}_{\mathrm{inf} / \text { letter }}=\frac{\mathrm{H}(\mathrm{S})}{\bar{\ell}}$ is get for interpretation of optimality of codes.
The results of these stages are given in Table 11. As previously presented, the optimality criteria for codes is $\mathrm{I}_{\mathrm{i} / \mathrm{s}} \rightarrow 1$. Obviously, it is seen from Table 11 that, binary codes constructed for each symbols of different alphabet by Fano-Huffman based statistical coding method is more optimal than Fano coding method and is as optimal as constructed by Huffman coding method but it is more easily applicable than Huffman coding method. Also, the improved coding method is more optimal than the others. Moreover, if a file is coded by Fano-Huffman based codes then the dimension of the file will be less than the files coded by the other considered coding methods. Hence, this means faster communication.

Table 11 Information per letter sent by constructed binary codes

\begin{tabular}{llllllll}
\hline $\begin{array}{l}\text { Source } \\
\text { Alphabet }\end{array}$ & $\begin{array}{l}\text { Ordinary } \\
\text { Codes } \\
\text { (bits) }\end{array}$ & $\begin{array}{l}\text { Shannon } \\
\text { Codes } \\
\text { (bits) }\end{array}$ & $\begin{array}{l}\text { Fano } \\
\text { Codes } \\
\text { (bits) }\end{array}$ & $\begin{array}{l}\text { Improved } \\
\text { Fano } \\
\text { Codes } \\
\text { (bits) }\end{array}$ & $\begin{array}{l}\text { Shannon } \\
\text { Fano } \\
\text { Elias } \\
\text { Codes } \\
\text { (bits) }\end{array}$ & $\begin{array}{l}\text { Huffman } \\
\text { Codes } \\
\text { (bits) }\end{array}$ & $\begin{array}{l}\text { Fano-Huffman } \\
\text { based Codes } \\
\text { (bits) }\end{array}$ \\
\hline English & 0.8145 & 0.8801 & 0.9834 & 0.9839 & 1.0792 & 0.9905 & 0.9888 \\
Turkish & 0.8732 & 0.9075 & 0.9937 & 0.9937 & 1.0955 & 0.9939 & 0.9939 \\
French & 0.7971 & 0.8885 & 0.9854 & 0.9854 & 1.0911 & 0.9899 & 0.9899 \\
German & 0.8190 & 0.9100 & 0.9901 & 0.9901 & 1.1083 & 0.9915 & 0.9901 \\
Spanish & 0.8032 & 0.9150 & 0.9909 & 0.9909 & 1.1161 & 0.9924 & 0.9916 \\
Russian & 0.8839 & 0.9085 & 0.9925 & 0.9936 & 1.2142 & 0.9936 & 0.9936 \\
\hline
\end{tabular}




\section{Conclusion}

It is seen that, binary codes constructed by FanoHuffman based statistical coding method carry information per letter as much as codes constructed by Huffman coding method. However, by this coding method the less subset you divide the more optimal codes you obtain. Thus, this result make Fano-Huffman based statistical coding method preferred coding methods as Huffman coding method for each of the considered languages. Fano-Huffman based statistical coding method takes less time than Huffman coding method to construct binary codes. However, it require more pure computation than Huffman coding method by means of dividing the source alphabet to subsets and this means faster coding.

As it is commonly known, operating system of computers based on American Standard Code for Information Interchange (ASCII) which is ordinary binary codes. Therefore, another main result from this study is the advantage of Fano-Huffman based codes rather than ASCII. Obviously, it can be concluded from this study that ordinary codes are not optimal because they have the highest average codeword length and the least information per letter. Hence, since ASCII codes are ordinary codes, the text coded by them will be larger in size contrary to Fano-Huffman based codes. So, ASCII codes are not preferred codes.

Consequently, Fano-Huffman based codes can be used in computer systems for data compression rather than ASCII for faster communication. Because, if a file is coded by Fano-Huffman based codes then the dimension of the file will be less than file coded by ASCII but it will transmit the same information by using codes consist of less bits.

\section{References}

Aazhang, B. (2004). http://cnx.rice.edu/content/m10176/latest/, Creative Commons.

Cover, T. M. \& Thomas, J. A. (1991). Elements of information theory. USA: John Wiley \& Sons, Inc.
Faller, N. (1973). An adaptive system for data compression. In 7th Asilomar conference on circuits, systems, and computers , 593-597.

Gallager, R. (1978). Variations on a theme by Huffman. IEEE Transactions on Information Theory, 24(6), 668-674.

Hankerson, D., Harris, G. A. \& Johnson, P. D. (2003). Introduction to information theory and data compression (2nd ed.). Boca Raton, FL: Chapman \& Hall/CRC Press.

Hankerson, D., Harris, G. \& Johnson, P. (1998). Introduction to information theory and data compression. CRC Press.

Huffman, D. (1952). A method for the construction of minimum redundancy codes. Proceedings of IRE, 40(9), 1098-1101.

Kieffer, J. C., \& Yang, E. (2000). Grammar-bassed codes: a new class of universal lossless source codes. IEEE Transactions on Information Theory, 46(3), 737-754.

Knuth, D. (1985). Dynamic Huffman coding. Journal of Algorithms, 6, 163-180.

Roman, S. (1997). Introduction to Coding and Information Theory. New York: Springer-Verlag.

Rueda, L. (2002). Advances in data compression and pattern recognition. $\mathrm{PhD}$ thesis, School of Computer Science, Carleton University, Ottawa, Canada.

Rueda, L. G. \& Oommen B. J. (2004). A Nearly-Optimal Fano-Based Coding Algorithm. Information Processing and Management, 40, 257-268.

Sayood, K. (2000). Introduction to data compression ( $2^{\text {nd }}$ ed.). Morgan Kaufmann.

Венцель, Е. С. (1969). Теория Вероятностей, Москва.

Pratt, F. (1939). Secret and urgent: The story of codes and ciphers. Blue Ribbon Books.

Phamdo, N. (2001). http://diwww.epfl.ch/mantra/CoursINF

OII/Web/compression/english.html, State University of New York.

Stephens, D. (2002). http://www.santacruzpl.org/readyref/files/g1/ltfrqsp.shtml, Santa Cruz Public Libraries, California. 
Shamilov A. and Yolacan S. (2005). Various binary codes for probability distribution of Turkish letters. International Conference Ordered Statistical Data: Approximations, Bounds and Characterizations, pp.70 Izmir, Turkey.

Shannon, C. E., \& Weaver, W. (1949). The mathematical theory of communications. University of Illinois Press.

Witten, I., Moffat, A. \& Bell, T. (1999). Managing gigabytes: Compressing and indexing documents and images (2nd ed.). Morgan Kaufmann.
Yolacan, S. (2005). Statistical properties of different languages based on entropy and information theory. Anadolu University Graduate School of Sciences, Master of Science Thesis (at turkish), Eskisehir.

Ziv, J. \& Lempel, A. (1977). A universal algorithm for sequential data compression. IEEE Transactions on Information Theory, 23(3), 337-343.

Ziv, J. \& Lempel, A. (1978). Compression of individual sequences via variable-rate coding. IEEE Transactions on Information Theory, 25(5), $530-536$. 